\title{
Online Transgender Health Information Seeking: Facilitators, Barriers, and Future Directions
}

\author{
Laima Augustaitis \\ laima@umich.edu \\ University of Michigan School of Information \\ Ann Arbor, Michigan \\ Kristi E. Gamarel \\ kgamarel@umich.edu \\ University of Michigan School of Public Health \\ Ann Arbor, Michigan
}

\author{
Leland A. Merrill \\ lelandm@umich.edu \\ Center for Sexuality and Health Disparities, University of \\ Michigan School of Nursing \\ Ann Arbor, Michigan \\ Oliver L. Haimson \\ haimson@umich.edu \\ University of Michigan School of Information \\ Ann Arbor, Michigan
}

\begin{abstract}
Transgender people face difficulties accessing healthcare from providers, and thus often turn to online sources to seek health information. However, online platforms may not properly support trans health information seeking, and health information found online may be limited in accuracy. To examine how online platforms can best support trans health information seeking, we conducted online focus groups with trans people $(n=26)$ about their experiences with online health information and their needs and desires for online health information seeking platforms. We found that trans people face both facilitators and barriers to finding accurate, actionable information online. Facilitators include online community discovery, group privacy features, and the dual synchronous and asynchronous nature of online content. Barriers include platform censorship, misinformation, hate speech, and lack of tools to flag inaccurate content. We provide recommendations for how platforms can support trans health information seeking by ensuring that medical information is accurate, accessible, easy to locate, and relevant to a diverse set of trans identities and experiences.
\end{abstract}

\section{CCS CONCEPTS}

- Human-centered computing $\rightarrow$ Empirical studies in collaborative and social computing.

\section{KEYWORDS}

online health information seeking, social media, health, online communities, misinformation, content moderation, privacy, transgender, nonbinary

ACM Reference Format:

Laima Augustaitis, Leland A. Merrill, Kristi E. Gamarel, and Oliver L. Haimson. 2021. Online Transgender Health Information Seeking: Facilitators,

Permission to make digital or hard copies of all or part of this work for personal or classroom use is granted without fee provided that copies are not made or distributed for profit or commercial advantage and that copies bear this notice and the full citation on the first page. Copyrights for components of this work owned by others than the author(s) must be honored. Abstracting with credit is permitted. To copy otherwise, or republish, to post on servers or to redistribute to lists, requires prior specific permission and/or a fee. Request permissions from permissions@acm.org.

CHI '21, May 8-13, 2021, Yokohama, Japan

(C) 2021 Copyright held by the owner/author(s). Publication rights licensed to ACM. ACM ISBN 978-1-4503-8096-6/21/05 ..\$15.00

https://doi.org/10.1145/3411764.3445091
Barriers, and Future Directions. In CHI Conference on Human Factors in Computing Systems (CHI '21), May 8-13, 2021, Yokohama, Japan. ACM, New York, NY, USA, 14 pages. https://doi.org/10.1145/3411764.3445091

\section{INTRODUCTION}

Transgender (defined as those whose current gender is different from that assigned at birth, including nonbinary people, hereafter shortened to "trans") individuals and communities disproportionately experience structural and social barriers to accessing healthcare, which contribute to significant health disparities [3, 40]. There is not enough information surrounding trans health needs due to a variety of factors, including trans people's erasure in data, trans people having limited access to health care, healthcare providers' inadequate knowledge, and limited research [51]. Moreover, trans people often delay seeking healthcare due to factors such as minority stress, traumatization in medical environments, and structural barriers such as cost and access to insurance [39]. For example, delaying care due to avoiding discrimination can contribute to poorer health outcomes for trans people [65]. Among trans people's information needs, health information has often been listed as a top priority [13].

Due to physical world barriers to trans health information seeking, trans people often access health information online [57]. Trans people rarely have a typical patient provider relationship with their healthcare providers, and often report needing to educate their providers about their needs $[40,58]$. Barriers to accessing trans information is not only due to stigma, but also a lack of existing information [57]. Trans people use a variety of in-person and virtual networks to acquire social support; online spaces are especially vital when in-person support networks are unavailable [24, 35]. People come to online communities when they need help, and they receive this help from similar others, and sometimes from health professionals and moderators [8]. For trans people in particular, online communities are places to reduce social isolation and experiment with trans identity [8,23]. Given pervasive structural and social barriers to accessing health care and comprehensive medical information, the Internet, particularly social media sites, may provide promise for trans people to access appropriate information by means of connecting with community.

The purpose of this research project was to explore the experiences of trans people accessing health information online, including 
how they use online platforms as tools to accomplish health information seeking tasks. To address these questions, we conducted three asynchronous online focus groups with 26 participants from across the United States. We contribute an understanding of which aspects of extant online platforms (i.e., social media platforms and user-generated information sites) are facilitators, and which are barriers to searching for health information. The main results substantiate and expand previous literature that discussed platformspecific policies that disproportionately disadvantage and exclude trans users [5, 28]. Drawing from topics salient to participants, we contribute a working definition of trans health. Lastly, based on our results, we provide several design recommendations to improve existing and future platforms to better accommodate trans users. This research informs ways forward to increase trans people's access to relevant health information in online formats and reduce the proliferation of inaccurate trans health information within online spaces. These contributions may extend beyond the trans context to increase understanding of health information access for a wide range of marginalized identities.

\section{RELATED WORK}

\subsection{Online Health Information Seeking}

Seeking health information online has long been studied among non-trans specific samples. According to a recent survey of mobile phone users internationally, about $60 \%$ of users reported using their mobile phone to search online for health information, surpassing other information seeking behaviors such as looking for political information [67]. It is estimated that around $72 \%$ of people online also use the Internet to search for health information [18]. There are many factors that influence using online sources for accessing health information. Johnson and Ambrose [41] argued that online health communities can fill important information gaps in the present health care system, which is especially useful for those isolated from others such as people in rural communities. Costello [9] described how patients sometimes felt dismissed or felt as though doctors could not answer some questions related to their health conditions. De Choudhury et al. [10] found that users reported seeking information online rather than in person due to convenience, privacy, and abundance of results for each search query. To a lesser extent, participants sometimes reported using online searching because they were unhappy with information they had received from providers. People seek health information online in many ways, such as via search engines, provider websites, and online health communities, and by utilizing in person or online networks through social media sites $[18,31]$.

People choose online health information sources (e.g., search engines, health communities, social media sites) due to factors including health literacy [70], illness type [7, 10], and health information seeking goals. Online health communities offer the unique benefit of connecting with others in similar situations such as survivors of traumatic health events [36], those living with chronic illnesses [48, 52], and caregivers of people with rare illnesses [38, 44]. Facebook groups for caregivers of children living with rare illnesses enabled participants to find others in similar geographic area, share firsthand advice, and disseminate resources and publications [38, 44]. Moreover, Oh et al. [54] reported that getting health support on Facebook was significantly associated with greater selfreported social support.

However, the benefits of online health information seeking come alongside many flaws. Sites and platforms are populated with inaccurate health information - both maliciously and innocently shared. The responsibility of eliminating misinformation can fall to online health community moderators and/or the platforms themselves, some of whom work to accurately identify and address misinformation [56]. For example, sometimes people without medical training moderate groups in which health information is posted. Moreover, some platforms are edited to inhibit some forms of misinformation (e.g., anti-vaccination campaigns) from gaining visibility [56]. Despite people's attempts to obtain accurate health information, the search result order can bias users into viewing questionable resources as credible [21]. People can be reluctant to share health information they found online with their health provider due to the stigma associated with online information searching [4].

Online platform affordances influence where people share some information versus others [47]. For example, an anonymized online platform such as Reddit may work well when searching for depression help, whereas Facebook may be used by the same person to share fitness data [47]. With a sample of people managing diabetes treatment, Newman et al. [52] found that people shared more personal information surrounding their health and struggles within a diabetes online health community, while they edited or did not share the same information with their Facebook networks.

Among non-trans specific LGBTQ+ online health information seeking research, published studies are largely focused on sexual health. Mitchell et al. [50] found that sexual minority youth were more than three times more likely to report using online resources to access sexual health information than heterosexual/straightidentified youth. Magee et al. [49] examined if and how LGBTQ+ youth seek sexual health information online, and found that the most prominent information seeking topic was HIV/STIs, and to a lesser extent interpersonal topics such as relationship advice. Even fewer studies specifically examine information searching needs of trans people; we review these next.

\subsection{Online Trans Health Information Seeking}

A small body of previous research has explored online trans health information topics, such as how trans people use online networks for health information seeking. Several researchers have found that social media platforms such as Twitter [42] and Tumblr [34] were used to voice trans health concerns, questions, needs, and thoughts. Karami et al. [42] systematically observed thousands of tweets from about 300 users and out of 125 topics, 54 topics were health-related. Hawkins and Gieseking [34] found that posts associated with the hashtags \#ftm (female to male) and \#mtf (male to female) commonly also included other hashtags about surgeries, hormones, and even doctors' names.

Qualitative works in the health information seeking/online health communities areas with a trans sample are relatively sparse. Hawkins and Haimson [35] found that transition blogs on Tumblr provided immense mental health support for a sample of trans users. Although not a study explicitly measuring health information seeking, Selkie et al. [66] reported that some members found 
medical advice from trans posts and users on social media. Liang et al. [46] explored trans youth's format and design preferences to receive sexual health information, and found that participants preferred alternate formats for digesting information depending on the information topic (e.g. relationships, sexually transmitted illnesses). However, sexual health information needs did not exist in isolation for participants because they brought up other health information needs such as medical gender affirmation. In Evans et al.'s [17] study, trans youth reported using a variety of online sources such as social support groups, search engines, and nonprofit organization websites. Motivators for using online sources included exploring trans identity and finding support groups [17]

Previous research scratched the surface in understanding some of the barriers trans people face when interacting with online information sources, such as misinformation and inaccessible information presentation. Evans et al. [17] described how trans youth frequently came across misinformation, such as resources about conversion therapy. Deutsch [11] searched the web using eight structured search strings related to trans health information, and found evidence of misinformation, and that many sources were relatively inaccessible. For instance, only $40 \%$ of the sites included accurate prescription information for hormones. Additionally, information was not presented in a bulleted, informative format, and lacked graphics. This could be a large barrier to engaging with health-related materials, and also poses accessibility concerns for users who may not be familiar with medical jargon. Furthermore, information can be inaccurate. Although trans people have healthrelated questions and concerns, accurate and consistent information is not yet available online. Other times, the information is there, but is not accessible to diverse learning styles.

Outside of a health context, it has been shown that trans users engage with online platforms in novel, unexpected ways to curate their identities on social media platforms. For example, Hanckel et al. [30] described that some trans participants used the nickname feature in Facebook Messenger to try out different preferred names - a use case its designers likely did not consider. Haimson et al. [25] and Hanckel et al. [30] described how trans users employ strategies for identity and information management, such as managing multiple accounts and adjusting privacy settings to hide posts from particular audiences. Horak [37] noted that YouTube enables trans vloggers to create videos showing their presentation change over time, yet these videos often center whiteness and gender norms.

Social media platforms include a wide range of barriers specific to trans users. In a non-health context, cumbersome content deletion processes, "real name" requirements, and one-profile policies have been shown to be inhibitory to trans users [5, 25, 28]. Additionally, online platforms can help foster trans communities, but also can be spaces of experiencing various forms of violence with privacy implications [26, 45, 62]. As trans health misinformation on online platforms, online harassment, and trans-inhibitory platform designs have become commonplace, we need further understanding of potential risks and barriers trans people face while seeking health information online. In the present research, we examine online platform affordances that serve as facilitators and barriers to trans online health information seeking.

\subsection{Previous LGBTQ+ Research in HCI}

Previous research in HCI has examined intersections of LGBTQ+ people and social technologies. LGBTQ+ users consider affordances and audiences when deciding which content to share on which sites $[12,22]$. Within LGBTQ+ spaces, people may experience discrimination for not fitting within a particular narrative of LGBTQ+ experience [71]. Previous LGBTQ+ HCI research has tackled topics such as minority stress [61], LGBTQ+ hate crime reporting [20], supporting LGBTQ+ parents [1], critiquing gender recognition technology which excludes trans experiences [29, 43, 63], LGBTQ+ people living in rural communities [32,33], safety for trans and/or nonbinary people of color [68], and LGBTQ+ social media use and identity $[14,23,62]$. However, to our knowledge, HCI researchers have not yet examined trans health online information seeking. LGBTQ+ identity as related to social technologies must be considered with attention to race, class, gender, sex, ability, and the intersections thereof $[16,53,64]$.

\subsection{Research Aims and Contributions}

Although there is little research published on trans health information seeking online, the existing body of literature supports that trans people often seek health information through online sources such as social media platforms. However, platform affordances related to accessing trans health information through online platforms have been under-studied. Thus, we lack vital knowledge about how social media and online resources may support or hinder trans health information seeking practices via their features, content, privacy affordances, and policies. Johnson and Ambrose [41] suggest that in order to realize online health communities' potential benefits, researchers must first discover "how" and "why" those online health communities are being used. Therefore, we contribute first steps in filling this research gap regarding facilitators and barriers to online trans information seeking using social media platforms and user-generated information sites. Additionally, we contribute implications for design of not only existing systems, but also potential future platforms such as a dedicated trans health platform. This work is essential to increase trans people's access to health information in the wake of widespread health disparities.

\section{METHODS}

We used an asynchronous online focus group methodology to address our research questions. This is a departure from past research on trans health information, which has used document review methods [34, 42] or semi-structured interviews [66]. Asynchronous focus groups have previously been used for health research with minority populations and are especially useful for discussions of sensitive topics [60]. We chose to use this method to foster flexibility and intergroup dialogue and minimize disruptions caused by time zone and participant schedule differences. This method also enabled participants to discuss potentially sensitive topics without having their real name or identity attached.

We recruited participants by posting recruitment flyers on Facebook, Twitter, Instagram, and Tumblr. The posts included hashtags relevant to trans communities, as well as some intersections thereof with racial and ethnic identities (e.g., \#transgénero [transgender in Spanish] and \#qtpoc [queer/trans people of color]). A total of 
257 individuals completed a screening survey that was linked from our recruitment flyer. The only eligibility criteria for participants was being trans and/or nonbinary and 18+ years of age. The screening survey offered space for participants to self-identify their gender, sexual orientation, race, ethnicity, age, geographical area, and (dis)ability.

Focus groups each occurred over the course of three days in August, November, and December 2019 on the online platform FocusGroupIt.com. Each focus group included 7-10 participants (3 focus groups, $n=26$ participants). We stopped after three focus groups because our emergent themes reached saturation after the third focus group. Our selection methods involved choosing participants from the survey responses to optimize diversity on 6 demographic dimensions: gender, race, ethnicity, age, (dis)ability status, and geographical area. We reached out to survey respondents via email individually to invite them to participate and obtained informed consent. None of the respondents were considered ineligible to participate; rather, some were not invited because their demographic was over-represented in the screening survey pool (e.g., young white people). Some respondents did not respond to the invitation email, and were replaced by others. Summary of participant demographics are included in Table 1. Each participant was given the choice to get their participant incentive payment through a virtual Amazon gift card or a physical prepaid MasterCard gift card for either an amount of $\$ 25$ to $\$ 40$ depending on the amount of questions in the focus group.

Focus group prompts approached topic areas such as 1) where participants access trans health information outside of providers, 2) facilitators and barriers to online trans health information seeking, and 3) potential features, layouts, and stakeholders to include for an ideal online trans health platform. Focus group prompts were slightly modified iteratively to increase clarity. The prompts used the words "trans health information" and asked about interactions in those contexts. We did not operationalize the term "trans health information" in aims to not bias responses towards any particular topics (e.g. access to providers, hormones).

FocusGroupIt.com was recommended by Reisner et al. [60] and included a number of affordances for both the focus group participants and moderator. Participants could either privately (only viewable by the moderator) or publicly (viewable to the moderator and the other participants in the same focus group) respond to each of the prompts, and they had flexibility in presenting themselves anonymously, pseudonymously, and with or without a profile picture of their choice. Participants often filled in their bio with more information about themselves to share with the group. FocusGroupIt.com allowed the moderator (first author) to schedule prompts and had all participants respond to each prompt sequentially in the same order. We staggered prompts across day and night to accommodate schedule and time zone differences. When prompts were posted, each participant received email notifications. Participants could view each others' responses, and could use threaded comments to respond to others' responses.

The moderator studied methods papers (e.g. [72]) in advance to prepare for navigating any potential issues that may have come up in the focus groups. The moderator remained available to answer questions via email throughout the duration of the study. Additionally, participants were informed that they could directly contact
Table 1: Participant Demographics

\begin{tabular}{lr}
\hline & $\begin{array}{r}\text { Percentage } \\
(n=26)\end{array}$ \\
\hline Gender & $30.8 \%$ \\
Transfeminine & $34.6 \%$ \\
Transmasculine & $34.6 \%$ \\
Nonbinary & \\
\hline Race/Ethnicity & $26.9 \%$ \\
Hispanic / Latino/a/e/x & $26.9 \%$ \\
White & $19.2 \%$ \\
Black / African American & $19.2 \%$ \\
Asian / Asian American & $19.2 \%$ \\
Mixed Race / Multiracial & $3.8 \%$ \\
Native American / Indigenous & \\
\hline Age & \\
Mean: 30.2 (SD = 8.4, range: 19-50) & $57.7 \%$ \\
\hline Disability Status & $42.3 \%$ \\
Reported having one or more disabilities & $30.8 \%$ \\
Reported not having disabilities & $30.8 \%$ \\
\hline Geographic Location in US & $15.4 \%$ \\
South & $15.4 \%$ \\
West & $7.7 \%$ \\
East & \\
Midwest & \\
Central & \\
\hline Participants could choose multiple gender and race/ethnicity \\
options, so percentages add up to greater than 100\%. \\
\hline
\end{tabular}

the PIs with any concerns about the study or moderator. Though we had these mechanisms in place, no issues arose.

The first author analyzed the transcripts first using an inductive open coding approach [6]. The first author met with the fourth author weekly to discuss codes, potential themes, and the data analysis process. Next, all authors met as a group to group codes into emergent themes [6]. The themes we chose to focus on in this paper are those most relevant to facilitators and barriers to online trans health information seeking. All study procedures were reviewed and deemed exempt by University of Michigan's Institutional Review Board (IRB).

\section{RESULTS}

\subsection{Focus Group Interactions}

Despite the asynchronicity of participant activity, participants utilized the threaded commenting feature to interact with each other's answers. Participants often agreed with one another, built on topics brought up by others, and offered affirmations. For example, Planned Parenthood was brought up several times and participants shared personal experiences and anecdotes about Planned Parenthood services and resources in the comments. As a whole participants were quite active and engaged with the focus groups: 25 out of 26 participants responded to all prompts and 99\% of prompts were answered by all 26 participants. 


\subsection{Trans Health Definition}

One contribution of this work is a definition of "trans health," based on the types of topics participants described when we asked them about trans health information seeking without first defining trans health. Throughout the focus groups, participants described their own trans health needs and drew from personal trans health information seeking experiences. Based on these responses, our working definition of "trans health" takes inspiration from the World Health Organization's holistic view of health: "physical, mental and social well-being," [55] in which one's gender identity and holistic needs are centered and affirmed. Determined individually, trans health may include, but is not limited to, a number of clinical and/or non-clinical gender affirmation processes (pronouns, name/gender marker changes, hormones, physical presentation, surgeries, etc.), access to affirmative healthcare, access to mental health resources, and equitable health insurance coverage.

\subsection{Seeking Health Information Outside of Providers}

When asked where they go to find online trans health information, participants listed a wide range of online information sources. Though focus group questions asked broadly about online information seeking, participants most frequently discussed social media platforms. Other online sources participants noted included online communities such as user-generated information sites (e.g., Quora, Reddit, Yelp) and specialized health provider websites such as Planned Parenthood and Callen Lorde (an LGBTQ+ health center in New York City). Additionally, participants mentioned using community-generated online resources such as the FTM Guide and Transbucket (a repository for post-surgery photos). Several participants also stated that they used physical world resources such as free clinics, local support groups, mental health providers, and LGBTQ+ non-profit organizations.

Overwhelmingly, participants listed one or more social media platforms or user-generated information sites as major sources of health information - from Facebook, Reddit, YouTube, Tumblr, and Instagram, to Discord (a chat platform initially for gamers, but now used in many contexts). Participants noted the benefit of connecting with people who could provide a firsthand account of the information. For example, P20 stated: "I trust personal experiences by trans people that have already went through what I am currently facing for the first time; even though everyone's experience is different, it helps give a general insight." P20's experience of trusting their peers for personal information was shared amongst many participants. Participants used social media for both medical and identity-related support.

Many participants noted the profound impact that online trans communities provided for their own identity formation, validation, and health, especially trans people in isolated areas. For example, P7 noted a lack of in person-resources:

Unfortunately, I don't have a lot of queer friends/resources immediately near me or in my life, so a lot of research around trans health info has been 1) from the internet and social media and 2) on my own. I go with my gut, ask questions prior to my visit, and just like above, if it doesn't work, start again.
Participants particularly emphasized that receiving support from other trans people, such as through word of mouth, was essential. For example, P2 said, "Having a recommendation from another trans person makes me the most comfortable." Similarly, other participants noted the opportunities social media platforms provide to return the favor by providing health information to other trans people. For instance, P14 stated, "The most joyful thing for me was connecting people with each other and with resources, and still is today." Participants' quotes demonstrate how impactful trans online communities can be for trans health information exchange, especially mental and social well-being. .

Not every participant said that they access health information from online sources. A subset of participants dissented, stating that they only trust medical journals or medical providers for healthrelated information: "I steer clear of social media as a resource for healthcare advice and stick to relevant articles from respected journals" (P24). This highlights a need for trans health information on social media to become more trustworthy and accurate.

Because most participants heavily favored social media sites and user-generated information platforms for health information seeking, the remaining Results sections predominantly focus on participants' reactions to these tools' performance for health information seeking purposes. Additionally, we include future design suggestions for online trans health information seeking.

\subsection{Facilitators to Online Health Information Seeking}

Aspects of social media platforms that facilitated trans health information seeking related to discovery mechanisms within social media groups, privacy features, and online platform content temporality.

4.4.1 Online Community Discovery. Participants appreciated the diverse abundance of community groups and resources continuously available on social media platforms and user-generated information sites. In P7's words, "I definitely, like a lot of people, rely on social media groups, mainly Twitter friends who are also queer and trans and occasionally, Reddit threads." Several noted that search features allowed for them to find unique, affirming groups at the intersection of several identities, such as "trans people with children" (P14). Being able to locate local trans people assisted with, for example, finding health provider location recommendations and hearing about experiences with surgeons:

I'm in a group called [name redacted] that has local meetups throughout the state and a Facebook group. It's great because it focuses on issues/people within the state, and it helps connect you to resources or people who can help you access those resources. (P23)

Participants' experiences highlight how online spaces can facilitate community connection, which is vital for trans health information seeking, including mental and social well-being. Online communities can be specific to narrow or broad audiences, such as trans-only Facebook groups and groups aimed to educate and integrate allies. While online community composition such as in trans Facebook 
groups allowed for a level of privacy, many times participants referenced privacy features as a facilitator for online trans communities and trans health information seeking, as we discuss next.

4.4.2 Social Media Group Privacy Features. Participants mentioned that privacy features such as private and hidden groups on sites like Facebook were helpful. As P26 noted, "Feeling safe is super necessary, there's some hate on Facebook and those private groups are great to open up." P19 stated that "I love joining private groups that are moderated by trans people and all members are trans." P19's quote mentions both the group composition and signals that privacy is key. Private groups only permit people who are in a Facebook group, rather than outsiders, to see the members in the group. This feature sometimes influenced participants' decisions of whether or not to join trans groups. When group affiliations are publicly listed, it can "out" people as trans to their entire network. Within groups, fostering favorable group composition with tools such as questioning prompts were highly commended by participants. For example, P21 noted that "groups having the ability to vet people via a questioning system make for the safest spaces you can find with the most first-hand experience from other trans gnc [gender non-conforming] people." Privacy features were important not only to moderate the members who join social media groups, but also helped some participants manage how their identity is visible to others across the platform.

4.4.3 Platform Content Temporality. The synchronous and asynchronous duality of online interactions made social media especially helpful for finding health information, according to participants. On social media, information is available in an archival format, but people can also interact in real-time via group dialogue and audience participation.

Synchronous social media capabilities such as posting questions and receiving feedback were helpful to participants seeking trans health information. One participant described this alwaysaccessible nature as such: "I like the fact that I can be actively involved or just hop on there if I need to connect with someone or want to be supportive" (P14). Synchronicity involved both interactions in online groups (e.g., trans support groups on Facebook) and features that allow for audience participation (e.g., the Question/Answer feature in Instagram stories as a method to gather input).

These same social media platforms often also provide asynchronous support for health information seeking. For instance, a participant mentioned that Instagram posts are permanent, enabling people to find trans health information that was posted in the past. Another mentioned how the archive of YouTube videos available helped them realize the abundance of online resources available: "I think YouTube has also helped with the proliferation of health info: seeing queer YouTubers definitely helped me realize how many resources there are out there" (P7). Although YouTube videos are not in real time, another participant pointed out their experience of watching YouTube videos as sometimes feeling more genuine. "In some ways using YouTube 'testimonials' [videos describing first-person trans experiences] feels like a more genuine representation of how folks are feeling about their bodies in real-time" (P11). Participants also described using social media to follow other trans people's experiences such as through photos of hormones effects or surgery results. Additionally, keyword searching within a group's conversation history was a helpful way to find relevant health information for their needs: "I like that I can search for individual words in Facebook groups" (P23). Social media sites' ability to both present and archive information were important for participants to find and share information.

\subsection{Barriers to Online Health Information Seeking}

Unfortunately, social media platforms also posed a number of barriers to fostering community connections among trans people seeking health information, including platform-initiated censorship, the presence of abundant misinformation and hate speech, and the lack of tools to flag false content.

4.5.1 Platform Censorship - Educational Posts and Identity Labels. Participants described being negatively impacted by unproductive censorship on platforms, especially platform-initiated censorship. Many participants brought up Tumblr's recently-updated posting policy banning all "adult" content, which inadvertently included medical and educational trans content (e.g., surgery photos) [27]. As P21 stated, "Tumblr lost a lot of its credibility from heavy moderating and non-friendly behavior towards trans educational posts." Platform policies that do not allow nudity in medical and educational contexts restrict trans health information sharing.

Platform-initiated censorship also included censoring people's identity expression via identity labels such as name and pronouns. Participants frequently commented negatively about name and pronoun labeling on social media sites. "Real name" [28] policies were barriers to joining trans health information seeking community spaces on Facebook. P23 stated, "I think it's important for trans people to not be booted from social media over dead names. That's a first step." "Deadnaming" means "referring to a transgender person by their birth name, even after they have specified their chosen name" (Michallon, 2019). Participants commented on bureaucratic name changing policies, remarking that these made it seem as though "Facebook was some government ID" (P21). P22 described,

I used to have a second Facebook account under an alternate name that I used to access transgender health groups, but Facebook deactivated it for suspecting me of having that second account that wasn't under my birth name. Despite my pleas they didn't give it back... the fact that I can only have one account under my birth name limits the amount of groups I am able to join, and also ensures I'm dead named within the trans groups that I'm in unfortunately.

Additionally, some participants talked about the fact that pronouns stay the same across the platform (e.g., a person cannot use different pronouns within a particular group than on their timeline), which forces some to choose non-representative pronouns to avoid being "outed" to their entire network.

4.5.2 Misinformation and Hate Speech. Although platforms were often quick to ban and remove trans content and users, these same platforms often allowed alarming levels of misinformation and hate speech. For example, P24 drew attention to the "abundance of false information or opinions of transphobic people that can sometimes 
drown out the important and truthful info." Participants reported receiving inaccurate information about topics such as hormones, mental health, binding (the action of making one's chest look flatter using clothing, binders, etc.), surgery, and coexisting conditions. For example, $\mathrm{P} 7$ described being told online that their trans identity was a result of a mental illness:

I was told - rather incorrectly - that my gender struggle (rather, my difficulty to understand what was feelings of dysphoria) was a dissociative symptom and meant I was bipolar and a danger to myself, but not nonbinary. Due to my decaying mental health, I believed this, felt instant guilt, all of which ultimately led to me delaying seeking aid for what is actually a dissociative disorder wholly unconnected from my gender.

This quote demonstrates the damaging personal consequences that can result from online trans health misinformation. Similarly, P4 was wrongly told online to not initiate hormones while being treated for anxiety medication. "I read that my anxiety would be exacerbated by HRT and that my anxiety medications wouldn't be as effective. This scared the shit out of me and contributed to my decision to stop medically transitioning." P16 reported finding inaccurate medical advice online about binding:

I remember before I had access to good binders there was all sorts of bad information about how to bind using ace bandages and duct tape and such. It was bad. I even ended up using an ace bandage at one point because I didn't realize how harmful it could be.

P16's experience highlights physical harm that can occur when one receives online misinformation about trans health. P18 mentioned seeing false information about gatekeeping, bottom surgeries, and puberty blockers, and noted:

I wish the people I heard this from were just being trolls but they were parents that bought into the scaretactic stories out there. Related to this are people getting puberty blockers and Hormone Replacement Therapy mixed up and that puberty blockers always create irreversible changes and infertility.

As these examples illustrate, trans health misinformation on social media can have particularly harmful physical and mental well-being effects, and can affect healthcare choices, such as deciding whether to pursue various medical gender affirming procedures.

Hate speech such as transphobia and trans shaming also occurred frequently on social media platforms. Transphobia and trans shaming can include many alternate ways of attempting to invalidate someone's trans identity or "othering" someone. For example, P20 ran across "a trans shaming video" about de-transitioning. Detransitioning videos outline experiences of trans people who have pursued gender affirming therapies then stopped and/or reverted therapies. Sometimes these videos are shared within groups or to trans individuals with the intention to falsely characterize being trans as a "phase." Social media also gives "transmedicalists" (i.e. "transmeds") platforms to spread hate speech and misinformation. Transmedicalists are trans people who believe that trans people who do not pursue medical transition are not trans. P21 gave the example of a harmful transmedicalist trans person with many social media followers: "[He] disvalues the experiences of people who either can't afford to transition to his level, who just don't pass, or who don't want to transition past a certain point or medically at all." Misinformation and hate speech as demonstrated by the participants' quotes above can be widely shared on social media platforms and cause substantial harm to trans individuals seeking health information online.

4.5.3 Lack of Tools to Flag Inaccurate Content and Sources. Participants described issues regarding a lack of member screening and content moderation that leads to high frequencies of transphobic hate speech and misinformation. Rather than platforms removing or flagging misinformation, participants often reported that intervening became the community members' responsibility. For example, one participant mentioned that they had seen other members within trans groups intervene and correct false information: "[I] have asked questions on Facebook groups and Reddit and received uninformed information from people - but it was essentially corrected by other people so there was no harm" (P3). P23 described a similar situation that had just happened the same day as our focus group:

This happened today. Someone was talking in a general LGBT group about how the effects of hormone therapy (for trans men) is reversible, and I had to jump in and say that's not the case and that communication between provider and patient is extremely important, as are realistic goals.

In cases like these, participants expressed desire to have agency in flagging information as false, such as with "a fact check score" (P18). The lack of mechanisms to fact check, or determine which information is credible, harms trust in platforms and hinders people's attempts to find actionable trans health information online. However, as noted in the two examples above, trans online community members are often resilient within these spaces despite abundant misinformation, such as by fact checking and correcting harmful misinformation.

\subsection{Ideal Online Trans Health Information Seeking Platform}

While there is not yet a dedicated social media site for accessing trans health information that includes content on physical, mental, and social well-being, we asked participants what features they would like to see in an ideal trans health information seeking platform. We asked about the general look and feel, any essential features, and which stakeholders to integrate in planning and sustaining a platform in the future. We asked participants about how a novel system might be designed to inspire creativity and thinking related to "future making" [15], a means to give marginalized populations a voice in how future technologies are designed and to elicit design recommendations according to their values [2]. A platform that employs our working definition of trans health (see section 4.2), which is drawn from participants' experiences, will also be useful to support well-being in which one's gender identity and holistic needs are centered and affirmed.

4.6.1 Look and Feel of Ideal Trans Health Information Seeking Platform. When participants referenced what they thought an ideal platform would look like, often they referred to existing platforms 
in their mental schema. For example, P7 referred to this ideal system as a blend of Tumblr, Twitter, and YouTube but with greater inclusivity for trans users. "I'd really something that blends Tumblr, Twitter, and YouTube, run by queer folks of all walks of life with a clear statement of inclusivity and actual, active moderation of hate speech, which a lot of platforms are actively... not doing" (P7). One participant mentioned structuring the site as a medical journal. Other themes were more abstract in nature and did not reference extant information sources. For example, participants often mentioned design choices that promote inclusivity and accessibility. Participants mentioned many times that the site should be cleanly designed, easy to navigate, and designed to not "out" people:

I think it should be very user friendly, to be inclusive for everyone including older individuals, or people that have disabilities. Make it easy to use, and an outlay that's not too flashy, more professional. Subtle enough that it's not going to out someone that hasn't maybe come out yet, like a few others have commented.

Additionally, inclusivity extended to include being traumainformed: "The information provided would be culturally informed, trauma-informed and accessible, both in an ability way and in an SES [socio-economic status] way" (P6). Trauma-informed resources are cognizant of past trauma people may have experienced that inform future experiences. A resource that talks about body parts with non-anatomical terms can be an example of a trauma-informed resource. Taken together, participants' suggestions point toward a platform that may have features that look and feel like existing, familiar social media platforms, but that would incorporate values important for trans health information seeking, such as inclusivity and community.

4.6.2 Features Available on an Ideal Trans Health Information Seeking Platform. Participants described the following features as important for an ideal trans health information seeking platform.

Question and Answer. Many participants suggested that an ideal platform should incorporate avenues for discussion. Namely, it was important for users to be able to ask their health questions relevant to their situations and receive responses from both trans people and healthcare experts. As $\mathrm{P} 18$ put it, "An 'Ask me anything' section where people with lived experience and various professionals can answer questions (without trying to replace individualized medical care)." A few participants suggested borrowing ideas from other platforms - Discord, YouTube, and the Kotex website. When P6 referenced the Kotex website as a good platform after which to model the Q\&A features, they suggested making it more gender inclusive and socially aware:

Weirdly enough, I think I would model it after the U by Kotex website, where people can ask questions and they provide an answer from a gyno [gynecologist], a mom and a peer. Ofc [of course] it would be different for a trans health website, maybe from GP/NP [General Practitioner/Nurse Practitioner] who is educated on serving trans folks, perhaps a social worker or something, and a trans person.

Both scientific credibility and individual experiences were important for information trust and validation. P9 said an ideal platform would "also verify information with current accepted medical practices and current/new reputable studies. Also offer current news relating to trans medical treatment and offer a forum for discussion. Others' experiences are still valuable." Participants saw strengths in presenting information with scientific input as well as the input from people with lived experiences relevant to the subject material.

Appropriate Identity Labels. Accessing trans health information on a platform in part depends on being able to accurately present one's identity on that platform. Participants posited platform suggestions that remedy the identity label deficits on other platforms, such as by providing more tailored gender, name, and pronoun options. For example, P21 said a platform should allow
more pronoun options for people who use neopronouns! Some people don't like using just the binary he/she, and having those locked to the most common sex for those pronouns isn't inclusive... Some people who are gnc [gender non-conforming], don't like binary pronouns or they/them, and would want an option available for it/its, xe/xim, and other neopronouns.

Participants emphasized again the importance of preferred names over dead names: "I also think it's important to allow people to use the correct name rather than their dead name" (P24). Additionally, it was important that names and pronouns could change throughout the site: "The ability to set a preferred name/pronouns inside of individual groups or visible to individual people of your choosing" (P22). These suggestions for an ideal platform address some of the barriers to accessing trans health information online that we described above.

User Account Authentication and Security. Privacy and security were salient topics in participants' suggestions for an ideal platform. To address user account types, people brought up verification processes for users of the sites. P8 stated, "I think as for access, possibly make an account and go through registration period first... before any posts can be viewed? This is mostly a precaution because of the internet, and trolls/harassers could potentially make people unsafe on the site." One person went on to say that the platform should be invite only: "I would say a private invite to it in order to screen out potentially dangerous/toxic individuals" (P20). Some participants mentioned implementing multiple types of accounts for different users, and an authentication process for healthcare providers on the platform: "I think there would be specific accounts for transgender people and clinicians. And there would be a verification process for the clinicians" (P24). For user accounts, several people stated the importance of users having a level of optional anonymity on the platform to conceal identifying information. They suggested protecting people's identities with the "ability to be confidential as not everyone has come out yet" (P9). Though they described several unique security processes, participants did not agree on any single process of administering account types on health information seeking platforms.

Content Moderation Balance. Participants discussed how an ideal trans health information platform needed to provide the right content moderation balance to reduce misinformation and allow for meaningful information exchange. An ideal platform would, in P8's words, ' not only squash hate speech but also promote healthy discussion." For several participants, presence of human moderators was important to filter out toxic individuals who are abusing the 
platform. P18 noted that "forums and chats with human moderators that can keep 'chasers' away who treat us as a fetish." Often, people mentioned the importance of signaling good and bad sources of information. "I feel that verification of content truthfulness should be at the forefront" (P24). Participants suggested implementing trustworthiness signals through platform features such as giving stars, scores, or badges. For example,

\section{It would be great if social media platforms had easily accessible names of groups and recommendations of those groups by stars so that people could vet them a bit before bothering to join especially since some of them are very toxic environment and that's the last thing we need when we have health needs, especially mental health needs. (P14)}

Participants suggested improving the content moderation balance by employing methods such as flagging posts to counter online misinformation and hate speech.

Information Accessibility and Inclusive Design. A consistent value among participants was that the platform be made available in multiple formats to be accessible to a wide range of users. For example, P21 suggested the platform be "mobile and desktop friendly," while P26 stated it should be both " $a$ website and an app to have easy access." P17 extended these suggestions to say that phone support should be available: "People would be able to access this info via the internet and also live phone support for mental health issues." P22 mentioned including video content for those who learn that way: "I like to get information via video content as opposed to reading, so maybe a little like YouTube but with a forum option." P5 suggested that in addition to being an online resource, some of the resources could potentially be available offline with "maybe a booklet of some kind, too." Format and information architecture features could provide opportunities to promote inclusion on trans health seeking platforms.

Beyond format of resources, participants emphasized inclusivity and intersectionality in the design of health information platforms. Suggestions to increase accessibility of information and its architecture included features to assist trans users with disabilities, because "inclusion is imperative" (P24). P11 stated that the platform "would be accessible for folks with screen readers. It would have a search bar that allows people to look up specific tags like (transition, HRT [hormone replacement therapy], gay, asexual, etc.)." Keyword searching and tagging came up frequently among participants. P2 wanted an ideal platform to include "tags lots of tags, afab [assigned female at birth], amab [assigned male at birth], nonbinary, hrt [hormone replacement therapy], surgery, etc. where you can narrow down what you are looking for." P4 referenced tagging features in terms of intersectionality, noting that "it would be able to allow patients to search by intersectionality, so we can search by class, ethnic identity and identity." Intersectionality as a guiding framework came up numerous times, such as when P24 described:

I also think it's important for us to be more intersectional in that one thing may work for someone whose only 'medical issue' is being trans but it could be ineffective or dangerous say for a transgender person with heart problems.
Taken together, participants' ideas provide insights for how to provide inclusive design across a wide range of trans identities and experiences.

4.6.3 Constituents for an Online Trans Health Information Seeking Platform. Including diverse perspectives was a consideration among participants for choosing whom to integrate into health information seeking platforms. According to participants, an ideal platform for trans health information seeking would include a blend of trans people, a wide array of health professionals and other professionals integral to trans health and well-being, and allies such as friends and family members.

Most frequently participants mentioned that the platform would include both trans people and health care providers and other specialists. In P4's words, the platform should include " $a$ wide network of specialists banding together as a network." Participants listed several key groups of professional stakeholders who should be involved in the planning and execution of a trans health seeking platform, including healthcare providers, mental health providers, and legal experts. Participants also mentioned integrating professionals working in social work ("mental health professionals such as social workers [and] medical professionals, including mental health" (P3)) or other medical professionals ("Trans friendly health providers, mental health providers and legal assistance would be a part of this site" (P17)). Additionally, P26 suggested including both beauty experts and trans activists into the platform: "Doctors, health experts, beauty experts, legal experts, and activists to talk about health systems and rights globally."

Participants also mentioned integrating family members and allies into the platform. P20 described including " a section for partners, friends and family too, but only if they're accepting," and P21 would include "trans-friendly allies who have made active efforts to improve the trans community/to learn for the betterment of their trans family or partners."

For participants, it was important that the platform be openly accessible to trans people, yet include different levels of authentication for non-trans members. This included ideas such as verification processes for providers ("vetted in doctors with experience on trans healthcare" (P21)) and only allowing allies who were accepting of trans identities.

\section{DISCUSSION}

The results of this qualitative study substantiate previous research literature outlining that trans users post and/or consume health information on social media platforms and user-generated information sites [17, 34, 42, 66]. Unlike several previous qualitative studies which have described how online spaces can help improve trans people's well-being $[35,62,66]$, our research questions were specific to the health information seeking behaviors among trans people online. Evans et al. [17] explicitly explored why people access health information online, and our results support their findings that trans users find community with other trans people within online social media networks and that trans users encounter substantial misinformation while seeking health information. Our research, to our knowledge, uniquely contributes an understanding of facilitators and barriers to online information seeking using social media platforms and user-generated information sites with trans users. 
Previous research has examined how social media platform affordances can facilitate online health information seeking in nontrans samples [10, 36, 52], and some of our results corroborate these studies; yet we find several important differences among trans populations. In the present study, facilitators to online health information seeking included social media groups and content temporality. Newman et al. [52] reported that people seeking diabetes assistance often preferred to share detailed emotional and health information among online health communities rather than Facebook because Facebook is more public. Our results differ because people spoke of Facebook primarily in the context of private Facebook groups, which they often used as online health communities. Facebook groups helped some participants access health information because the groups were identity-specific, and some utilized privacy mechanisms such as screening questionnaires. Additionally, the abundant access to information, archived posts, and social networks at any time of day was also beneficial, supporting Hinson [36]. Participants chose to find health information online especially due to the community aspect for seeking and providing advice to others. This differs from [10] where social support was the least frequent motivator (among the sample's self-reported motivators) to access health information using social media. These differences highlight the importance of peer support among trans online communities.

We describe how, unfortunately, trans people face unique barriers in online health information seeking - multiple aspects of social media platforms were prohibitive for health information seeking within our sample. Participants reported that unnecessary platforminitiated censorship of trans educational posts concurrently exist in environments where there are no clear indicators of which information is reliable. For example, platforms censored valid trans health information as "adult" content, prohibited people from being able to join trans social media groups due to concerns of being "outed," and imposed barriers such as "real name" and one-account policies. These and similar policies have previously been shown as barriers to participation for trans users online [5, 28], and we expand these results in a trans health context. Lastly, participants reported a large presence of misinformation, shared both innocently and maliciously by both trans people and others (e.g., parents), and hate speech. While Scheuerman et al. [62] briefly described misinformation as a type of "outsider harm," given the prevalence of inaccurate information shared within trans online health communities, we must consider misinformation also as part of what Scheuerman et al. [62] called "insider harm."

Next, we describe some ways new and existing platforms can be designed to facilitate and reduce barriers to trans online health information seeking.

\subsection{Implications for Design}

This research takes first steps in suggesting design recommendations for a dedicated trans health information seeking platform. We describe principles, based on our results, for how such a platform could be designed. It is not known whether improving current systems, creating a new platform, or a hybrid is best, but our results and design implications provide potential future directions for any of these. Regardless of platform, policies that exclude or negatively impact trans people will be detrimental to trans health and well-being. Because many of our results reference faults in extant platforms, we clearly articulate opportunities for extant platforms to improve experiences for trans users seeking health information.

5.1.1 Design for Diverse Identities and Experiences. A trans health information platform must not forefront any singular narrative of trans experience. Instead, it should be designed in consideration of the trans population's wide array of health needs, such as mental health needs, accessibility needs, the presence of preexisting conditions, and the unique challenges faced by trans people of color. Importantly, participants noted that health advice that works for some trans people can be dangerous for others, so a platform must indicate these risks and provide ways to search for information given one's particular health conditions and needs. Additionally, information must be presented in an accessible format, to reduce disabled people's barriers to engaging with health information.

5.1.2 Include Vetted Contributors. A trans health platform, according to participants, should integrate many groups in addition to trans people such as health providers, social workers, activists, legal advisors, and allies. However, participants often provided a caveat that participants such as non-trans allies and health providers should be carefully screened before being allowed to participate in community discourse.

5.1.3 Tailored Content Moderation. Participants provided suggestions for content moderation to remedy deficits present on other platforms they used to access health information. For example, participants suggested omitting "real name" policies and implementing platform-specific tools to flag misinformation. Because participants described often needing to conduct moderation themselves in trans online health communities, platforms should enable communitybased moderation procedures (following from recommendations in [26]).

5.1.4 Present Health Information in Parallel with Lived Experiences. It was important for participants that a trans online health platform provide opportunities for community dialogue and presenting information in parallel with firsthand accounts of experiences. This suggestion aligns with trans communities' increased caution towards medical establishments and reliance on community narratives and experiences for health information [19, 59, 69]. Participants suggested implementing a Question and Answer feature, in which both trans people and healthcare providers could weigh in and contribute their expertise in a format that facilitates easy comparison of the two.

5.1.5 Make Health Information Easy to Locate. In existing platforms, participants valued the synchronous and asynchronous duality of information, especially when it allows for archival searching and information cataloguing via tags and drop downs. Information should also be provided in multiple formats (e.g., videos, graphics, text) to increase information's accessibility.

5.1.6 Next Steps. We have detailed features that an ideal trans health information platform should employ, and who should be included in such a space. Though there are existing platforms where trans health information is circulated, the unique barriers of platform-initiated censorship, misinformation, and hate speech make clear the need for new design. Because few of the platforms 
mentioned by participants were designed with trans health information seeking in mind, our study asked participants to consider how a more intentional design could function. A team of designers and researchers could build such a platform by implementing these recommendations. Design suggestions could also be implemented in existing platforms to improve trans health information seeking for users. Future research would be needed to understand how a dedicated trans online health information platform is used in practice, and to further iterate on its design. Additionally, future research may explore feasibility and adoptability of a standalone platform as compared to refining existing platforms, as well as health outcomes related to online trans health information seeking behaviors (e.g., source, frequency).

\subsection{Ethical Implications}

Due to the potentially sensitive nature of the topics participants discussed in the focus groups, we were ethically obligated to determine in advance how to respond if participants posted distressing content The research team was prepared to refer participants to resources (e.g., Trans Lifeline) if distressing incidents had occurred, and to remove content that may negatively impact other participants. As in Reisner et al. [60], focus group participants provided social support to each other around negative experiences, so it is possible that focus groups positively impacted participants. When conducting research on trans health information behaviors, researchers must be prepared to connect participants to resources and support if needed, and to heavily moderate discussion.

\subsection{Strengths and Limitations}

This research presented both strengths and limitations. The sample was diverse across many identities, and many participants held multiple marginalized identities. The sampling technique was a strength because the responses included people from all over the US and across multiple generations. Future work could conduct similar studies in non-US contexts. Additionally, the online focus group research design was highly beneficial in bringing into discussion a diverse group of people living in different time zones and with variable schedules. However, these data represent a selection bias for trans users who are already adopters of social media platforms and results are not generalizable to all trans people. Additionally, participants that self-reported as Indigenous and/or Native American were disproportionately underrepresented among other racial/ethnic identities in the sample. The recruitment flyer mentioned both health information and online information which may have oversampled people who already search for health information online. The focus group platform allowed for participants to make their posts viewable to the entire group or to only the moderator. Despite this feature, there may have still been a social desirability bias in the focus groups for members to answer in a manner that would be viewed positively by others. Though the focus groups generated substantial discussion among participants, due to the textual format we were unable to pick up on cues present in inperson focus groups and follow up accordingly. Moreover, it was sometimes unclear whether participants were describing interactions on social media with people in their known network or with strangers; provisions should be made in future studies to minimize such ambiguity.

\subsection{Key Takeaways}

Through this work, we have uncovered key takeaways that, if seriously considered in existing or new platforms' designs, will improve online trans health information seeking processes.

(1) As stated in our working definition (see section 4.2), trans health involves not only physical well-being, but also mental and social well-being.

(2) Trans health information is frequently exchanged in online communities.

(3) Enabling trans people to easily find and join online trans health communities is vital.

(4) In trans health online communities, privacy features must work to maintain safe information exchange and prohibit unwanted audiences.

(5) Online trans health information should be available both synchronously and asynchronously.

(6) Platform policies often hinder users from accessing trans health information.

(7) Misinformation and hate speech are serious barriers to trans health information seeking, and an important area for platform improvements.

(8) One way to address misinformation is to enable users to flag inaccurate content and sources.

\section{CONCLUSION}

The results of this research suggest that social media platforms serve an unconventional purpose to provide important health information for trans populations, albeit each platform is abundant with tradeoffs. Trans users exemplify resilience in curating spaces for disseminating trans health information seeking while navigating barriers such as hate speech and misinformation. It is essential that online platforms become more inclusive to the experiences and needs of trans users. For example, reducing unnecessary barriers such as legal name policies and increasing users' ability to moderate problematic content are necessary to ensure that online trans health communities can thrive. As trans health needs and linguistic norms related to trans identities evolve rapidly among trans communities, ongoing community engagement is essential for designers of social media platforms and user-generated information sites.

Further research may explore design and adoptability of a dedicated trans health information seeking platform by integrating some of these findings. For example, researchers can explore experiences using a prototype online health platform with communities. Future research can also provide platform-specific recommendations to extant social media platforms. As Hanckel et al. [30] points out, platform affordances may be used by trans users especially to foster community connection and navigate boundaries. Therefore, when significant barriers to information seeking surface, designers must adapt. Social media platforms are commonly used for spreading news articles, sales of items, and many other possibilities that were not available in early iterations. Aspects such as the proliferation of trans health misinformation and inhibitory platform policies negatively affect the health seeking process of trans users. 
Therefore, small changes to platforms, such as the elimination of policies that disproportionately negatively impact trans people, may contribute to better health outcomes. However, for the long term, the relationship between trans communities and the medical field may be further remedied by engaging with communities to create a dedicated spaces, online and in person, that blend real person testimonials in parallel with provider advice. Over time, this platform can help dispel misinformation online and facilitate the process for finding accurate and relevant health information. As participants pointed out, it is essential that this work is not completed through a singular perspective. This work should be cooperative and aimed at reducing structural and interpersonal barriers to accessing appropriate information and care.

Although transgender people have many health-related questions and concerns, accurate and consistent information is not abundant or accessible online [57]. Therefore, it is essential that researchers further explore not only how trans people search for and access health information online, but also how to ensure that accurate medical information is accessible to the population it impacts and how online spaces can be more inclusive to the more marginalized subsets of the diverse trans population (e.g., trans people of color; trans people with particular health conditions). Once barriers are mitigated, online platforms can be a vital way forward for trans health information seeking.

\section{ACKNOWLEDGMENTS}

We would like to thank all focus group participants for sharing their experiences and expertise. Thanks to Shanna Kattari for help with participant recruitment and study design. We also acknowledge University of Michigan's MCubed Program for funding this research.

\section{REFERENCES}

[1] Lindsay Blackwell, Jean Hardy, Tawfiq Ammari, Tiffany Veinot, Cliff Lampe, and Sarita Schoenebeck. 2016. LGBT Parents and Social Media: Advocacy, Privacy, and Disclosure During Shifting Social Movements. In Proceedings of the $2016 \mathrm{CHI}$ Conference on Human Factors in Computing Systems (CHI '16). ACM, New York, NY, USA, 610-622. https://doi.org/10.1145/2858036.2858342

[2] Alan Borning and Michael Muller. 2012. Next steps for value sensitive design. In Proceedings of the 2012 ACM annual conference on Human Factors in Computing Systems - CHI '12. ACM Press, Austin, Texas, USA, 1125. https://doi.org/10.1145/ 2207676.2208560

[3] Judith Bradford, Sari L. Reisner, Julie A. Honnold, and Jessica Xavier. 2012. Experiences of Transgender-Related Discrimination and Implications for Health: Results From the Virginia Transgender Health Initiative Study. American fournal of Public Health 103, 10 (Nov. 2012), 1820-1829. https://doi.org/10.2105/AJPH.2012.300796 Publisher: American Public Health Association.

[4] Lauren Bussey and Elizabeth Sillence. 2017. (How) do People Negotiate Online Information into their Decision Making with Healthcare Professionals?. In Proceedings of the 2017 International Conference on Digital Health (DH '17). Association for Computing Machinery, New York, NY, USA, 1-5. https://doi.org/10. $1145 / 3079452.3079495$

[5] Matthew Carrasco and Andruid Kerne. 2018. Queer Visibility: Supporting LGBT+ Selective Visibility on Social Media. In Proceedings of the 2018 CHI Conference on Human Factors in Computing Systems (CHI '18). ACM, New York, NY, USA, 250:1-250:12. https://doi.org/10.1145/3173574.3173824

[6] Kathy Charmaz. 2006. Constructing Grounded Theory: A Practical Guide Through Qualitative Analysis. SAGE Publications.

[7] Yu Chi, Daqing He, Shuguang Han, and Jie Jiang. 2018. What Sources to Rely on: Laypeople's Source Selection in Online Health Information Seeking. In Proceedings of the 2018 Conference on Human Information Interaction \& Retrieval (CHIIR '18). Association for Computing Machinery, New York, NY, USA, 233-236. https://doi.org/10.1145/3176349.3176881

[8] Sabrina Cipolletta, Riccardo Votadoro, and Elena Faccio. 2017. Online support for transgender people: an analysis of forums and social networks. Health \& Social
Care in the Community 25, 5 (Sept. 2017), 1542-1551. https://doi.org/10.1111/ hsc. 12448

[9] Kaitlin L. Costello. 2016. Impact of patient-provider communication on online health information behaviors in chronic illness. Proceedings of the Association for Information Science and Technology 53, 1 (2016), 1-10. https://doi.org/10.1002/pra2.2016.14505301060_eprint: https://asistdl.onlinelibrary.wiley.com/doi/pdf/10.1002/pra2.2016.14505301060.

[10] Munmun De Choudhury, Meredith Ringel Morris, and Ryen W. White. 2014 Seeking and sharing health information online: comparing search engines and social media. In Proceedings of the SIGCHI Conference on Human Factors in Computing Systems (CHI '14). Association for Computing Machinery, New York, NY, USA, 1365-1376. https://doi.org/10.1145/2556288.2557214

[11] Madeline B. Deutsch. 2016. Evaluation of Patient-Oriented, Internet-Based Information on Gender-Affirming Hormone Treatments. LGBT Health 3, 3 (June 2016), 200-207. https://doi.org/10.1089/lgbt.2015.0116

[12] Michael Ann Devito, Ashley Marie Walker, and Jeremy Birnholtz. 2018. "Too Gay for Facebook": Presenting LGBTQ+ Identity Throughout the Personal Social Media Ecosystem. Proceedings of the ACM Human Computer Interaction (PACM) 2, CSCW (2018), 23

[13] Aubri A. Drake and Arlene Bielefield. 2017. Equitable access: Information seeking behavior, information needs, and necessary library accommodations for transgender patrons. Library \& Information Science Research 39, 3 (July 2017), 160-168. https://doi.org/10.1016/j.lisr.2017.06.002

[14] Brianna Dym, Jed R. Brubaker, Casey Fiesler, and Bryan Semaan. 2019. "Coming Out Okay": Community Narratives for LGBTQ Identity Recovery Work. Proc. ACM Hum.-Comput. Interact. 3, CSCW (Nov. 2019), 154:1-154:28. https://doi. org $/ 10.1145 / 3359256$

[15] Pelle Ehn, Elisabet M. Nilsson, and Richard Topgaard. 2014. Making Futures. https://mitpress.mit.edu/books/making-futures

[16] Sheena Erete, Aarti Israni, and Tawanna Dillahunt. 2018. An intersectional approach to designing in the margins. Interactions 25, 3 (April 2018), 66-69. https://doi.org/10.1145/3194349

[17] Yolanda N. Evans, Samantha J. Gridley, Julia Crouch, Alicia Wang, Megan A. Moreno, Kym Ahrens, and David J. Breland. 2017. Understanding Online Resource Use by Transgender Youth and Caregivers: A Qualitative Study. Transgender Health 2, 1 (Aug. 2017), 129-139. https://doi.org/10.1089/trgh.2017.0011

[18] Susannah Fox. 2014. The social life of health information. https://www. pewresearch.org/fact-tank/2014/01/15/the-social-life-of-health-information/

[19] Spencer Garrison. 2018. On the Limits of "Trans Enough": Authenticating Trans Identity Narratives. Gender \& Society 32, 5 (Oct. 2018), 613-637. https://doi.org/ $10.1177 / 0891243218780299$

[20] Cally Gatehouse, Matthew Wood, Jo Briggs, James Pickles, and Shaun Lawson. 2018. Troubling Vulnerability: Designing with LGBT Young People's Ambivalence Towards Hate Crime Reporting. In Proceedings of the 2018 CHI Conference on Human Factors in Computing Systems (CHI '18). ACM, New York, NY, USA, 109:1109:13. https://doi.org/10.1145/3173574.3173683

[21] Amira Ghenai, Mark D. Smucker, and Charles L.A. Clarke. 2020. A Think-Aloud Study to Understand Factors Affecting Online Health Search. In Proceedings of the 2020 Conference on Human Information Interaction and Retrieval (CHIIR '20). Association for Computing Machinery, New York, NY, USA, 273-282. https: //doi.org/10.1145/3343413.3377961

[22] Oliver L. Haimson. 2018. Social Media as Social Transition Machinery. Proc. ACM Hum.-Comput. Interact. 2, CSCW (Nov. 2018), 63:1-63:27. https://doi.org/ $10.1145 / 3274332$

[23] Oliver L. Haimson, Anne E. Bowser, Edward F. Melcer, and Elizabeth F. Churchill. 2015. Online Inspiration and Exploration for Identity Reinvention. In Proceedings of the 33rd Annual ACM Conference on Human Factors in Computing Systems (CHI '15). ACM, New York, NY, USA, 3809-3818. https://doi.org/10.1145/2702123. 2702270

[24] Oliver L. Haimson, Jed R. Brubaker, Lynn Dombrowski, and Gillian R. Hayes. 2015. Disclosure, Stress, and Support During Gender Transition on Facebook. In Proceedings of the 18th ACM Conference on Computer Supported Cooperative Work \& Social Computing (CSCW'15). ACM, New York, NY, USA, 1176-1190. https://doi.org/10.1145/2675133.2675152

[25] Oliver L. Haimson, Jed R. Brubaker, Lynn Dombrowski, and Gillian R. Hayes. 2016. Digital Footprints and Changing Networks During Online Identity Transitions. In Proceedings of the 2016 CHI Conference on Human Factors in Computing Systems (CHI '16). ACM, New York, NY, USA, 2895-2907. https://doi.org/10.1145/2858036. 2858136

[26] Oliver L Haimson, Justin Buss, Zu Weinger, Denny L Starks, Dykee Gorrell, and Briar Sweetbriar Baron. 2020. Trans Time: Safety, Privacy, and Content Warnings on a Transgender-Specific Social Media Site. Proc. ACM Hum.-Comput. Interact. 4, CSCW (2020), 27.

[27] Oliver L. Haimson, Avery Dame-Griff, Elias Capello, and Zahari Richter. 2019. Tumblr was a trans technology: the meaning, importance, history, and future of trans technologies. Feminist Media Studies (Oct. 2019), 1-17. https://doi.org/10. $1080 / 14680777.2019 .1678505$ 
[28] Oliver L. Haimson and Anna Lauren Hoffmann. 2016. Constructing and enforcing "authentic" identity online: Facebook, real names, and non-normative identities. First Monday 21, 6 (June 2016). https://doi.org/10.5210/fm.v21i6.6791

[29] Foad Hamidi, Morgan Klaus Scheuerman, and Stacy M. Branham. 2018. Gender Recognition or Gender Reductionism?: The Social Implications of Embedded Gender Recognition Systems. In Proceedings of the 2018 CHI Conference on Human Factors in Computing Systems (CHI '18). ACM, New York, NY, USA, 8:1-8:13. https://doi.org/10.1145/3173574.3173582

[30] Benjamin Hanckel, Son Vivienne, Paul Byron, Brady Robards, and Brendan Churchill. 2019. 'That's not necessarily for them': LGBTIQ+ young people, social media platform affordances and identity curation. Media, Culture \& Society (May 2019), 0163443719846612. https://doi.org/10.1177/0163443719846612

[31] Derek L Hansen, Holly A Derry, Paul J Resnick, and Caroline R Richardson. 2003 Adolescents Searching for Health Information on the Internet: An Observational Study. Fournal of Medical Internet Research 5, 4 (Oct. 2003), e25. https://doi.org/ 10.2196/jmir.5.4.e25

[32] Jean Hardy and Silvia Lindtner. 2017. Constructing a Desiring User: Discourse, Rurality, and Design in Location-Based Social Networks. In Proceedings of the 2017 ACM Conference on Computer Supported Cooperative Work and Social Computing (CSCW' 17). ACM, New York, NY, USA, 13-25. https://doi.org/10.1145/2998181. 2998347

[33] Jean Hardy and Stefani Vargas. 2019. Participatory Design and the Future of Rural LGBTQ Communities. In Companion Publication of the 2019 on Designing Interactive Systems Conference 2019 Companion - DIS '19 Companion. ACM Press, San Diego, CA, USA, 195-199. https://doi.org/10.1145/3301019.3323894

[34] Blake Hawkins and Jack Giesking. 2017. Seeking ways to our transgender bodies, by ourselves: Rationalizing transgender-specific health information behaviors Proceedings of the Association for Information Science and Technology 54, 1 (Jan. 2017), 702-704. https://doi.org/10.1002/pra2.2017.14505401122

[35] Blake W. Hawkins and Oliver Haimson. 2018. Building an Online Community of Care: Tumblr Use by Transgender Individuals. In Proceedings of the 4th Conference on Gender \& IT (GenderIT '18). ACM, New York, NY, USA, 75-77. https://doi org $/ 10.1145 / 3196839.3196853$

[36] Katrina L. Hinson. 2017. Framing illness through Facebook enabled online support groups. Communication Design Quarterly 4, 2b (March 2017), 22-31. https://doi.org/10.1145/3068755.3068758

[37] Laura Horak. 2014. Trans on YouTube Intimacy, Visibility, Temporality. TSQ Transgender Studies Quarterly 1, 4 (Nov. 2014), 572-585. https://doi.org/10.1215/ 23289252-2815255

[38] Maia Jacobs, Galina Gheihman, Krzysztof Z. Gajos, and Anoopum S. Gupta. 2019 "I think we know more than our doctors": How Primary Caregivers Manage Care Teams with Limited Disease-related Expertise. Proceedings of the ACM on Human-Computer Interaction 3, CSCW (Nov. 2019), 159:1-159:22. https //doi.org/10.1145/3359261

[39] Kim D. Jaffee, Deirdre A. Shires, and Daphna Stroumsa. 2016. Discrimination and Delayed Health Care Among Transgender Women and Men: Implications for Improving Medical Education and Health Care Delivery. Medical Care 54, 11 (Nov. 2016), 1010-1016. https://doi.org/10.1097/MLR.0000000000000583

[40] Sandy E. James, Jody L. Herman, Susan Rankin, Mara Keisling, Lisa Mottet, and Ma'ayan Anafi. 2016. The Report of the 2015 U.S. Transgender Survey. Technical Report. National Center for Transgender Equality, Washington, DC. http://www. transequality.org/sites/default/files/docs/USTS-Full-Report-FINAL.PDF

[41] Grace J. Johnson and Paul J. Ambrose. 2006. Neo-tribes: the power and potential of online communities in health care. Commun. ACM 49, 1 (Jan. 2006), 107-113. https://doi.org/10.1145/1107458.1107463

[42] Amir Karami, Frank Webb, and Vanessa L. Kitzie. 2018. Characterizing Transgender Health Issues in Twitter. In Proceedings of the Association for Information Science and Technology. http://arxiv.org/abs/1808.06022 arXiv: 1808.06022 .

[43] Os Keyes. 2018. The Misgendering Machines: Trans/HCI Implications of Automatic Gender Recognition. Proceedings of the ACM on Human-Computer Interaction 2, CSCW (Nov. 2018), 88. https://doi.org/10.1145/3274357

[44] Roderick Lamar Lee and Lynette M. Kvasny. 2014. Understanding the role of social media in online health: A global perspective on online social support. First Monday (2014). https://doi.org/10.5210/fm.v19i1.4048

[45] Ada Lerner, Helen Yuxun He, Anna Kawakami, Silvia Catherine Zeamer, and Roberto Hoyle. 2020. Privacy and Activism in the Transgender Community. In Proceedings of the 2020 CHI Conference on Human Factors in Computing Systems (CHI '20). Association for Computing Machinery, Honolulu, HI, USA, 1-13. https: //doi.org/10.1145/3313831.3376339

[46] Calvin A. Liang, Katie Albertson, Florence Williams, David Inwards-Breland, Sean A. Munson, Julie A. Kientz, and Kym Ahrens. 2020. Designing an online sex education resource for gender-diverse youth. In Proceedings of the Interaction Design and Children Conference (IDC '20). Association for Computing Machinery, London, United Kingdom, 108-120. https://doi.org/10.1145/3392063.3394404

[47] Yuting Liao. 2019. Sharing Personal Health Information on Social Media: Balancing Self-presentation and Privacy. In Proceedings of the 10th International Conference on Social Media and Society (SMSociety '19). Association for Computing Machinery, New York, NY, USA, 194-204. https://doi.org/10.1145/3328529.3328560
[48] Haley MacLeod, Kim Oakes, Danika Geisler, Kay Connelly, and Katie Siek. 2015. Rare World: Towards Technology for Rare Diseases. In Proceedings of the 33rd Annual ACM Conference on Human Factors in Computing Systems - CHI '15. ACM Press, Seoul, Republic of Korea, 1145-1154. https://doi.org/10.1145/2702123. 2702494

[49] Joshua C. Magee, Louisa Bigelow, Samantha DeHaan, and Brian S. Mustanski. 2012. Sexual Health Information Seeking Online A Mixed-Methods Study Among Lesbian, Gay, Bisexual, and Transgender Young People. Health Education \& Behavior 39, 3 (June 2012), 276-289. https://doi.org/10.1177/1090198111401384

[50] Kimberly J. Mitchell, Michele L. Ybarra, Josephine D. Korchmaros, and Joseph G. Kosciw. 2014. Accessing sexual health information online: use, motivations and consequences for youth with different sexual orientations. Health Education Research 29, 1 (Feb. 2014), 147-157. https://doi.org/10.1093/her/cyt071 Publisher: Oxford Academic.

[51] Viviane Namaste. 2000. Invisible Lives: The Erasure of Transsexual and Transgendered People. University of Chicago Press. Google-Books-ID: Pq5jwRVbvY8C.

[52] Mark W. Newman, Debra Lauterbach, Sean A. Munson, Paul Resnick, and Margaret E. Morris. 2011. It's not that i don't have problems, i'm just not putting them on facebook: challenges and opportunities in using online social networks for health. In Proceedings of the ACM 2011 conference on Computer supported cooperative work (CSCW' 11). ACM, New York, NY, USA, 341-350. https://doi.org/10.1145/1958824.1958876

[53] Ihudiya Finda Ogbonnaya-Ogburu, Angela D. R. Smith, Alexandra To, and Kentaro Toyama. 2020. Critical Race Theory for HCI. In Proceedings of ACM CHI Conference on Human Factors in Computing Systems. 16.

[54] Hyun Jung Oh, Carolyn Lauckner, Jan Boehmer, Ryan Fewins-Bliss, and Kang Li. 2013. Facebooking for health: An examination into the solicitation and effects of health-related social support on social networking sites. Computers in Human Behavior 29, 5 (Sept. 2013), 2072-2080. https://doi.org/10.1016/j.chb.2013.04.017

[55] World Health Organization. 2021. Constitution. https://www.who.int/about/ who-we-are/constitution

[56] Sherry Pagoto, Molly E. Waring, and Ran Xu. 2019. A Call for a Public Health Agenda for Social Media Research. Journal of Medical Internet Research 21, 12 (2019), e16661. https://doi.org/10.2196/16661 Company: Journal of Medical Internet Research Distributor: Journal of Medical Internet Research Institution: Journal of Medical Internet Research Label: Journal of Medical Internet Research Publisher: JMIR Publications Inc., Toronto, Canada.

[57] Aira Maria Pohjanen and Terttu Anna Maarit Kortelainen. 2015. Transgender information behaviour. Journal of Documentation 72, 1 (Dec. 2015), 172-190. https://doi.org/10.1108/JD-04-2015-0043

[58] Tonia Poteat, Danielle German, and Deanna Kerrigan. 2013. Managing uncertainty: A grounded theory of stigma in transgender health care encounters. Social Science \& Medicine 84 (May 2013), 22-29. https://doi.org/10.1016/j.socscimed. 2013.02.019

[59] Jay Prosser. 1998. Second Skins: The Body Narratives of Transsexuality. Columbia University Press.

[60] Sari L. Reisner, Renee K. Randazzo, Jaclyn M. White Hughto, Sarah Peitzmeier, L. Zachary DuBois, Dana J. Pardee, Elliot Marrow, Sarah McLean, and Jennifer Potter. 2018. Sensitive Health Topics With Underserved Patient Populations: Methodological Considerations for Online Focus Group Discussions. Qualitative Health Research 28, 10 (Aug. 2018), 1658-1673. https://doi.org/10.1177/ 1049732317705355

[61] Koustuv Saha, Sang Chan Kim, Manikanta D. Reddy, Albert J. Carter, Eva Sharma, Oliver L. Haimson, and Munmun De Choudhury. 2019. The Language of LGBTQ+ Minority Stress Experiences on Social Media. Proceedings of the ACM on HumanComputer Interaction 3, CSCW (Nov. 2019), 89:1-89:22. https://doi.org/10.1145/ 3361108

[62] Morgan Klaus Scheuerman, Stacy M. Branham, and Foad Hamidi. 2018. Safe Spaces and Safe Places: Unpacking Technology-Mediated Experiences of Safety and Harm with Transgender People. Proc. ACM Hum.-Comput. Interact. 2, CSCW (Nov. 2018), 155:1-155:27. https://doi.org/10.1145/3274424

[63] Morgan Klaus Scheuerman, Jacob M. Paul, and Jed R. Brubaker. 2019. How Computers See Gender: An Evaluation of Gender Classification in Commercial Facial Analysis and Image Labeling Services. Proceedings of the ACM on HumanComputer Interaction 3, CSCW (Nov. 2019), 33. https://dl.acm.org/doi/abs/10. $1145 / 3359246$

[64] Ari Schlesinger, W. Keith Edwards, and Rebecca E. Grinter. 2017. Intersectional HCI: Engaging Identity through Gender, Race, and Class. Proceedings of CHI (2017). https://pdfs.semanticscholar.org/27b4/ 9a706d907c5d6858068ba97bbe873fcf687f.pdf

[65] Kristie L. Seelman, Matthew J.P. Colón-Diaz, Rebecca H. LeCroix, Marik XavierBrier, and Leonardo Kattari. 2017. Transgender Noninclusive Healthcare and Delaying Care Because of Fear: Connections to General Health and Mental Health Among Transgender Adults. Transgender Health 2, 1 (Feb. 2017), 17-28. https://doi.org/10.1089/trgh.2016.0024

[66] Ellen Selkie, Victoria Adkins, Ellie Masters, Anita Bajpai, and Daniel Shumer. 2019. Transgender Adolescents' Uses of Social Media for Social Support. Fournal of Adolescent Health (Nov. 2019). https://doi.org/10.1016/j.jadohealth.2019.08.011 
[67] Laura Silver and Christine Huang. 2019. Social activities, information seeking on subjects like health and education top the list of mobile activities. https://www.pewresearch.org/internet/2019/08/22/social-activitiesinformation-seeking-on-subjects-like-health-and-education-top-the-list-ofmobile-activities/

[68] Denny L. Starks, Tawanna Dillahunt, and Oliver L. Haimson. 2019. Designing Technology to Support Safety for Transgender Women \& Non-Binary People of Color. In Companion Publication of the 2019 Designing Interactive Systems Conference (DIS '19 Companion). ACM, New York, NY, USA, 289-294. https: //doi.org/10.1145/3301019.3323898 event-place: San Diego, CA, USA.

[69] Allucquère Rosanne (Sandy) Stone. 1987. The Empire Strikes Back: A Posttranssexual Manifesto. http://sandystone.com/empire-strikes-back.pdf

[70] Yalin Sun and Yan Zhang. 2016. Individual Differences and Online Health Information Source Selection. In Proceedings of the 2016 ACM on Conference on Human Information Interaction and Retrieval (CHIIR '16). Association for Computing Machinery, New York, NY, USA, 321-324. https://doi.org/10.1145/2854946.2854989
[71] Ashley Marie Walker and Michael Ann DeVito. 2020. "'More gay' fits in better": Intracommunity Power Dynamics and Harms in Online LGBTQ+ Spaces. In Proceedings of the 2020 CHI Conference on Human Factors in Computing Systems (CHI '20). Association for Computing Machinery, Honolulu, HI, USA, 1-15. https: //doi.org/10.1145/3313831.3376497

[72] Andrea L. Wirtz, Erin E. Cooney, Aeysha Chaudhry, Sari L. Reisner, and American Cohort To Study HIV Acquisition Among Transgender Women (LITE). 2019. Computer-Mediated Communication to Facilitate Synchronous Online Focus Group Discussions: Feasibility Study for Qualitative HIV Research Among Transgender Women Across the United States. Fournal of Medical Internet Research 21, 3 (2019), e12569. https://doi.org/10.2196/12569 Company: Journal of Medical Internet Research Distributor: Journal of Medical Internet Research Institution: Journal of Medical Internet Research Label: Journal of Medical Internet Research Publisher: JMIR Publications Inc., Toronto, Canada. 